\title{
Análise epidemiológica da mortalidade por causas externas em Sobral, Ceará, no período de 2013 a 2017
}

\author{
Epidemiological analysis of mortality from external causes in Sobral, Ceará, from 2013 to \\ 2017
}

Análisis epidemiológico de la mortalidad por causas externas en Sobral, Ceará, en el período 2013-2017

Walter Oliveira Rios Junior ${ }^{1 *}$, José Francisco Igor Siqueira Ferreira ${ }^{1}$, Victor Carneiro de Souza ${ }^{1}$, Karyne Gomes Cajazeiras ${ }^{1}$, João Vitor Lopes Montes ${ }^{1}$, José Lucas Martins Costa ${ }^{1}$, Augusto Cesar Silva Vasconcelos Filho', Brunno Alexander Oliveira1, Madelline Alves Vasconcelos², Ulli Aguiar Vasconcelos ${ }^{2}$.

\section{RESUMO}

Objetivo: Descrever e analisar o perfil epidemiológico da mortalidade por causas externas (CE) no município de Sobral, Ceará, no período de 2013 a 2017. Métodos: Estudo descritivo, ecológico e de caráter quantitativo. Foram utilizados dados fornecidos pelo Ministério da Saúde, através do Sistema de Informações sobre Mortalidade (SIM), através dos quais, foi traçado o perfil epidemiológico da mortalidade por CE no município, bem como sua evolução durante o quinquênio analisado. Resultados: Houve o aumento da taxa de mortalidade por CE, no período analisado, de 99,16 para 107,53 óbitos por 100 mil habitantes. Os grupos mais acometidos foram homens (87,19\%), pardos (86,89\%), entre 20 e 39 anos (47,45\%). A principal causa externa de mortalidade foi o homicídio (49,25\%), sendo o meio mais empregado a arma de fogo $(75,2 \%)$, seguido dos acidentes de transportes (28,33\%), principalmente de motocicletas $(49,12 \%)$. Conclusão: $O$ estudo revela o progressivo aumento do número de mortes por causas externas no município. Além disso, o grupo mais acometido é o homem, jovem e pardo: perfil marginalizado pela sociedade. Nota-se, portanto, a necessidade do desenvolvimento não só de políticas de saúde, mas também de políticas sociais e de segurança pública a fim de minimizar o problema.

Palavras-chave: Epidemiologia, Mortalidade, Causas externas.

\section{ABSTRACT}

Objective: To describe and to analyze the epidemiological profile of mortality from external causes $(E C)$ in the municipality of Sobral, Ceará, from 2013 to 2017. Methods: Descriptive, ecological and quantitative study. Data provided by the Ministry of Health, through the Mortality Information System, through which the epidemiological profile of EC mortality in the municipality was traced, as well as its evolution during the fiveyear period analyzed. Results: There was an increase in the mortality rate by $\mathrm{EC}$, in the period, from 99,16 to 107,53 deaths per 100,000 habitants. The most affected groups were men $(87,19 \%)$, brown $(86,89 \%)$, between 20 and 39 years $(47,45 \%)$. The main external cause of mortality was homicide $(49,25 \%)$, with firearms $(75,2 \%)$, followed by transport accidents $(28.33 \%)$, mainly motorcycles $(49.12 \%)$. Conclusion: The study reveals the progressive increase in the number of deaths from external causes in the municipality. In addition, the group most affected is the man, young and brown: a marginalized profile by society. There is a need to develop not only health policies, but also social and public security policies in order to reduce the problem.

Key words: Epidemiology, Mortality, External causes.

${ }^{1}$ Universidade Federal do Ceará (UFC), Sobral - CE. *E-mail: walteroriosjr@gmail.com

2 Centro Universitário INTA (UNINTA), Sobral - CE.

SUBMETIDO EM: 5/2020

ACEITO EM: 6/2020

PUBLICADO EM: $8 / 2020$

REAS/EJCH | Vol.12(9) | e3893 | DOI: https://doi.org/10.25248/reas.e3893.2020 Página 1 de 8 


\section{RESUMEN}

Objetivo: Describir y analizar el perfil epidemiológico de la mortalidad por causas externas (CE) en el municipio de Sobral, Ceará, en el período de 2013 a 2017. Métodos: Estudio descriptivo, ecológico y de carácter cuantitativo. Se utilizaron datos del Ministerio de Salud, a través del Sistema de Informaciones sobre Mortalidad, a través de los cuales, se trazó el perfil epidemiológico de la mortalidad por CE en el municipio, así como su evolución durante el quinquenio analizado. Resultados: Hubo un aumento de la tasa de mortalidad por CE, en el período analizado, de 99,16 para 107,53 óbitos por 100 mil habitantes. Los grupos más afectados fueron hombres (87,19\%), pardos (86,89\%), entre 20 y 39 años $(47,45 \%)$. La principal causa externa de mortalidad fue el homicidio $(49,25 \%)$, siendo el medio más empleado el arma de fuego $(75,2 \%)$, seguido por los accidentes de transporte $(28,33 \%)$, principalmente de motocicletas $(49,12 \%)$. Conclusión: El estudio revela el progresivo aumento del número de muertes por causas externas en el municipio. Además, el grupo más afectado es el hombre, joven y pardo: perfil marginado por la sociedad. Así, se observa la necesidad de desarrollar no sólo políticas sanitarias, sino también políticas sociales y de seguridad pública para minimizar el problema.

Palabras clave: Epidemiología, Mortalidad, Causas externas.

\section{INTRODUÇÃO}

Segundo a Classificação Estatística Internacional de Doenças e Problemas Relacionados à Saúde - 10a Revisão (CID-10) as causas externas (CE) englobam traumas ou lesões decorrentes de causas acidentais ou intencionais. As causas externas são divididas em cinco grandes grupos: acidentes de transportes; suicídios; homicídios, incluídas as intervenções legais; causas de intenção indeterminada; e demais causas externas. As mortes por CE podem ser denominadas como óbitos "não naturais", oriundos de ações voluntárias, a exemplo dos casos de suicídio e homicídio, como também causas por intervenções repentinas e violentas, como aquelas provenientes de acidentes de trânsito (MODESTO JB, et al., 2019).

No Brasil, as causas externas consistem em um problema de saúde pública, com uma urgente necessidade do seu estudo e prevenção, demonstradas pelas crescentes taxas de incidência, prevalência e mortalidade. O Sistema Único de Saúde (SUS) é o ponto de convergência de todas as causas, sendo o principal meio buscado pelas vítimas para tratamento ou reabilitação (MESSIAS MM, et al., 2018).

A importância do tema não é de exclusividade nacional. De acordo com a Organização Mundial da Saúde (OMS), o aumento das causas de morte por acidentes e violência tem ocorrido em diversas regiões no mundo e correspondem a cerca de 5 milhões de mortes por ano, o equivalente a $9 \%$ da mortalidade mundial, esses elevados índices refletem no sistema de saúde, o que gera milhares de hospitalizações, atendimentos ambulatoriais e em emergências.

Em 2016, a principal causa de morte por CE no mundo foram os acidentes de trânsito, ocupando o oitavo lugar das principais causas de morte no mundo. Além disso, os acidentes de trânsito não aparecem no ranking de morte dos países desenvolvidos, sendo mais comum nos países emergentes que naqueles classificados como subdesenvolvidos. O desenvolvimento de estratégias mundiais para a redução de óbitos por causas externas, como a "Década de Ação para a Segurança no Trânsito (2011 a 2020)", pela Organização das Nações Unidas, em 2010, reafirmam a situação como um problema mundial (WHO, 2018).

Além disso, as causas externas causam um importante impacto aos cofres públicos. Somente em 2014, por exemplo, os gastos gerados por acidentes de trânsito à sociedade chegaram a $R \$ 12,3$ bilhões, sendo $\mathrm{R} \$ 8$ bilhões decorrente de gastos de saúde com a vítima e perda de produção de pessoas (LIMA TF, et al., 2019).

A conceituação de transição epidemiológica é fundamental no contexto de causas externas, na qual, há um modificação dos padrões de saúde e doenças que atingem a população, deixando de haver o predomínio de doenças infecciosas e parasitárias e destacando o crescimento da mortalidade por causas degenerativas e por causas externas (TAVARES J, 2018), com destaque para os homicídios e acidentes de trânsito (PREIS LC, et al., 2018). 
No Brasil, as CE foram responsáveis por $12,9 \%$ dos óbitos, configurando-se como a terceira maior causa de mortes no país. Dentre as CE, os homicídios foram responsáveis por $37,1 \%$ das causas de óbitos, os acidentes de transporte por $30,3 \%$ e suicídios por $6,8 \%$. Os $26 \%$ restante se dividiram entre outras causas, como por exemplo, os afogamentos (CORASSA RB, et al., 2017).

Mesmo com os possíveis casos de subnotificação e erros de classificação (atribuição de outras causas de morte) e a sensibilidade em torno do assunto, o suicídio representa a segunda principal causa de morte de pessoas entre 15 e 29 anos. Sendo, portanto, mais um problema de saúde pública mundial (SILVA, 2018).

Em alguns estados, como no Rio de Janeiro, uma integração de dados dos setores de saúde, segurança pública e imprensa possibilitou uma melhoria no registro de mortes por causas externas (LOPES AS, et al., 2018). Em relação às causas específicas os acidentes de trânsito, homicídios, suicídios, quedas e lesões intencionais ou acidentais aparecem como os principais causadores de mortes externas no Brasil e, juntos, ocupam a segunda posição dos óbitos totais no país (MESSIAS MM, et al., 2018).

Em razão da escassez de artigos que abordem o perfil epidemiológico da mortalidade por causas externas no município de Sobral - CE e a magnitude do tema nos âmbitos de saúde e social, ressalta-se a importância desse estudo no fornecimento de dados e como estratégia para identificação de fatores de risco e elaboração de futuras políticas públicas de intervenção.

\section{MÉTODOS}

Trata-se de um estudo descritivo, documental e de caráter quantitativo. Foram utilizados dados fornecidos pelo Ministério da Saúde, através do Sistema de Informações sobre Mortalidade (SIM) para a realização de um estudo abordando todos os casos de óbitos por causas externas no município de Sobral, Ceará, ocorridos no período de janeiro de 2013 a dezembro de 2017.

A origem dos dados disponibilizados pelo SIM é coletada por meio das Declarações de Óbitos (DO), com base na causa básica informada pelo médico atestante do óbito. Os dados de cada ano foram atualizados e revisados nos meses de agosto do ano seguinte, segundo o SIM. Foram coletados os dados referentes aos óbitos ocorridos no município de Sobral no período de 2013 a 2017 que apresentaram como causa de morte, algum dos óbitos expressos no capítulo XX do CID-10 (Causas externas de morbidade e mortalidade), sob os códigos presentes entre V01 e Y98. As variáveis obtidas para a análise foram: faixa etária, raça e sexo dos indivíduos.

Para melhor análise, os óbitos foram classificados em cinco grandes grupos: acidentes de transportes; suicídios; homicídios, incluídas as intervenções legais; causas de intenção indeterminada; e demais causas externas. A divisão se baseou respectivamente nos seguintes grupos do CID-10: V01-V99; X60-X84; X85Y09 e Y35-Y36; Y10-Y34; e demais códigos de V01-Y98. Através desses dados, o perfil epidemiológico da mortalidade por causas externas foi delineado no município, bem como sua evolução durante o quinquênio analisado pelo estudo. Para o cálculo das taxas de mortalidade, foram usadas as projeções anuais para a população, de acordo com o Instituto Brasileiro de Geografia e Estatística (IBGE), divulgadas em documento elaborado pelo Instituto de Pesquisa e Estratégia Econômica do Ceará (IPECE).

O cenário do estudo é o município de Sobral. O município está situado na região Noroeste do Ceará, a 235 quilômetros de Fortaleza, limita-se ao norte com os municípios de Massapê, Santana do Acaraú e a Serra da Meruoca, ao sul com Santa Quitéria, Groaíras e Cariré, ao leste com Itapipoca, Irauçuba e Canindé, e a oeste com Coreaú, Mucambo e Alcântara. Sobral é o segundo município mais desenvolvido do estado do Ceará e possui clima tropical, quente e seco com temperatura média de $30^{\circ} \mathrm{C}$.

Os dados obtidos foram tabulados através do software Microsoft Office Excel $2013 \AA$ e analisados por meio da estatística descritiva, por meio de cálculos de frequência absoluta e relativa. A taxa de mortalidade por causas externas foi obtida por meio da razão entre o número de óbitos por grupo de causa ocorrido e a população estimada para o município em cada ano analisado, sendo apresentados por 100 mil habitantes. $O$ mesmo se aplicou ao cálculo da taxa de mortalidade para o estado do Ceará, a região Nordeste e o Brasil. Os resultados obtidos estão apresentados no formato de gráficos e tabelas.

REAS/EJCH | Vol.12(9) | e3893 | DOI: https://doi.org/10.25248/reas.e3893.2020 Página 3 de 8 
Quanto aos aspectos éticos, o estudo está embasado na resolução 466/2012 do Conselho Nacional de Saúde (CNS) e, portanto, prima por seus princípios fundamentais: não maleficência, beneficência, autonomia e justiça. Todos os dados coletados têm acesso irrestrito e disponível pela rede mundial de computadores, sendo, portanto, de domínio público. A apreciação do projeto por um comitê de ético e pesquisa (CEP) foi dispensada, em razão dos meios consultados não conterem informações sigilosas, sendo disponíveis para acesso público.

\section{RESULTADOS E DISCUSSÃO}

A partir da coleta de dados, obteve-se um total de 5837 óbitos de indivíduos residentes no município de Sobral, no período de 2013 a 2017. Deste total, 3387 (58,03\%) foram de pessoas do sexo masculino, 2449 $(41,95 \%)$ de indivíduos do sexo feminino e, em apenas 1 caso $(0,02 \%)$, o sexo não foi especificado. Dos 5837 óbitos, $999(17,11 \%)$ foram decorrentes de causas externas. No período analisado, o ano com o maior número de óbitos por causas externas foi 2015 , com um total de 223 (22,32\%), um valor $42,95 \%$ maior que o ano com menor número de casos, 2016, cujo total de óbitos foi de 156 (15,61\%). O ano de 2017 aparece como o de segunda maior mortalidade, com um total de $221(22,12 \%)$ óbitos.

Em relação ao sexo, os homens aparecem como o grupo mais acometido, com um total de $871(87,19 \%)$ óbitos por causas externas. Já o sexo feminino é responsável por uma menor parcela, totalizando 128 $(12,81 \%)$ casos. A taxa de mortalidade por causas externas em Sobral, no ano de 2017, foi de 95,36 óbitos por 100 mil habitantes no sexo masculino e 12,16 no sexo feminino. Essa expressiva desigualdade de gênero já havia sido retratada no estudo à cerca da mortalidade por causas externas no Brasil realizado em 2010 realizado por (MOURA EC, et al., 2010). Resultados semelhantes foram encontrados nos estudos realizados em São Paulo, no período de 2008 a 2011 (GONSAGA RAT, et al., 2012), na Região Sul, no período de 2004 a 2013 (PREIS LC, et al., 2018), em Diamantina - MG, de 2001 a 2012 (CORASSA RB, et al., 2017).

Acredita-se que essa desigualdade está relacionada ao comportamento social de risco da população do sexo masculino, uma vez que, esse grupo comumente se envolve em agressões e discussões, agem com imprudência no trânsito, além de que, ao se comparar com as mulheres, consomem álcool em maior quantidade (VASCONCELOS SM, 2015).

No que se refere à faixa etária, evidenciou-se que a maior parte dos óbitos foi de pessoas jovens, com idade entre 20 e 39 anos, seguida por pessoas com idade entre 40 e 59 anos, representando $47,45 \%$ e $21,32 \%$. Em relação aos óbitos por causas externas em idosos, ocorreu um total de 124 óbitos nesse grupo, correspondendo a $12,41 \%$ dos casos.

Grande parte dos estudos apresentou resultados semelhantes, trazendo os jovens com idade entre $20 \mathrm{e}$ 39 anos o principal grupo acometido por óbitos por causas externas, como aqueles realizados na Região Sul, no período de 2004 a 20135, no Brasil em 201017 e em Diamantina-MG, de 2001 a 2012 (CORASSA RB, et al., 2017). Este resultado pode estar relacionado à imprudência resultante, neste grupo, do desejo de conhecer e testar seus limites, movidos pela sensação de liberdade e coragem desenvolvida nessa fase da vida (ASCARI RA, et al., 2013).

No que tange à cor/raça, observou-se predomínio de casos em pessoas pardas, com $868(86,89 \%)$ óbitos por causas externas, seguido de brancas, com 109 (10,91\%) casos. A cor/raça preta aparece com menor ocorrência, apresentando apenas 4 casos $(0,4 \%)$. Em $18(1,8 \%)$ casos, essa informação não foi especificada. Acredita-se que este resultado se justifique pelo fato da população com menor nível socioeconômico ser composta por pardos, sendo este grupo mais suscetível à violência social e à marginalização.

Quanto ao local de ocorrência, houve prevalência dos óbitos ocorridos em hospital e em via pública, representando, respectivamente, $43,74 \%$ e $31,23 \%$ dos casos. Apenas $9,9 \%$ dos óbitos ocorreram no próprio domicílio dos indivíduos.

Observa-se, conforme o Gráfico 1, que, no panorama geral de mortalidade por causas externas, houve predomínio, ao longo de todo o período, de óbitos por agressões (homicídios), com um total de 492 (49,25\%) casos. O ano de 2017 apresentou o maior número de óbitos por essa causa, totalizando $120(24,39 \%)$ casos, número consideravelmente superior à média anual calculada de 98,4 $\pm 20,79$ (Gráfico 1). 
Gráfico 1 - Perfil da mortalidade por causas externas em Sobral, CE no período de 2013 a 2017 (números absolutos).

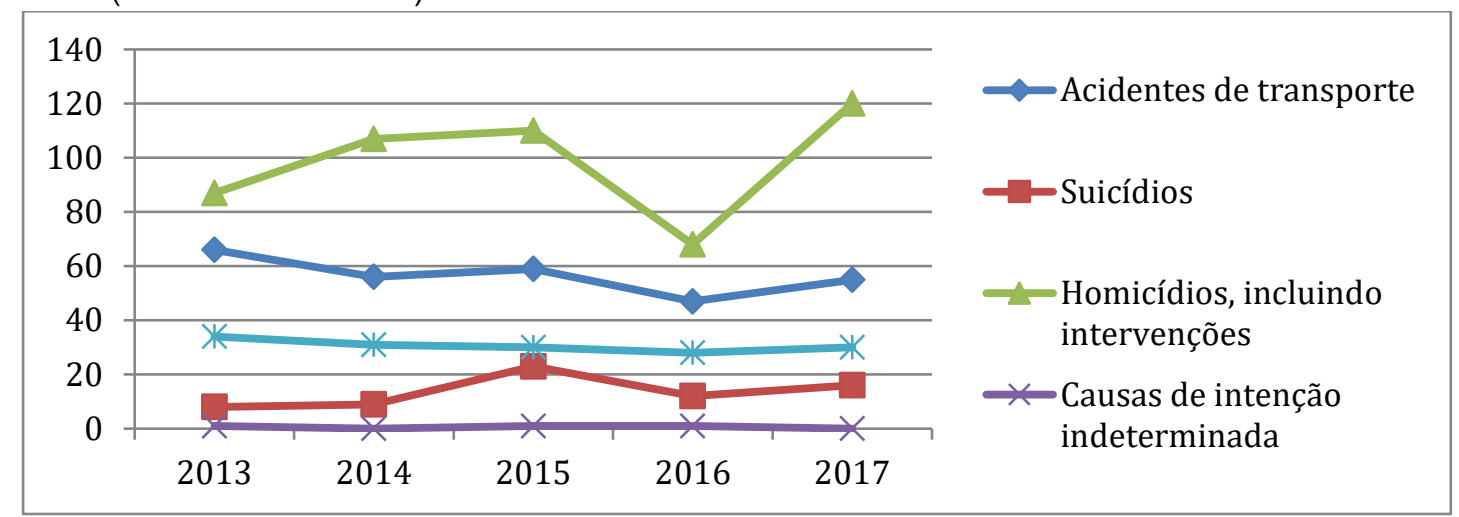

Fonte: Rios Junior WO, et al., 2020. Dados extraídos do Sistema de Informações sobre Mortalidade (SIM).

Esse crescimento expressivo no número de homicídios também foi evidenciado em estudos realizados na Região Sul, no ano de 2004 a 2013 (PREIS LC, et al., 2018), e em Curitiba-PR, de 1996 a 2011 (BARRETO MS, et al., 2016). Esse resultado é reflexo da violência social, relacionada à desigualdade social, e da fragilidade da segurança pública em todo o país (MELO GBT, et al., 2015).

Em relação às demais causas externas, observa-se pouca oscilação ao longo de todo o período analisado. Os óbitos por acidentes de transporte surgem como a segunda maior causa, com um total de $283(28,33 \%)$ casos, seguida pelas demais causas externas, que correspondem às outras formas de acidentes e àquelas não especificadas, com um total de $153(15,31 \%)$. Os suicídios aparecem com causa de $6,8 \%$ dos casos. Em todo período, apenas $3(0,3 \%)$ casos foram classificados como causas de intenção indeterminada.

No município de Sobral houve uma taxa de mortalidade por causas externas expressivamente maior, em ambos os anos analisados, que as encontradas na região Nordeste e no Brasil, como um todo. A Taxa de Mortalidade encontrada no município no ano de 2013 foi de 99,16 óbitos por 100 mil habitantes, enquanto no ano de 2017 foi de 107,53 óbitos por 100 mil habitantes (Tabela 1).

Tabela 1 - Taxa de Mortalidade (por 100 mil habitantes) por grupos de causas, dos anos de $2013 \mathrm{e}$ 2017, no município de Sobral, no estado do Ceará, na região Nordeste e no Brasil, 2018.

\begin{tabular}{|c|c|c|c|c|}
\hline Grupo de causas & Sobral & Ceará & Nordeste & Brasil \\
\hline \multicolumn{5}{|l|}{ Total } \\
\hline 2013 & 99,16 & 103,44 & 86,07 & 75,45 \\
\hline 2017 & 107,53 & 108,5 & 91,58 & 75,19 \\
\hline \multicolumn{5}{|c|}{ Acidentes de trânsito } \\
\hline 2013 & 33,39 & 27,97 & 23,34 & 21,61 \\
\hline 2017 & 26,76 & 22,31 & 18,96 & 16,92 \\
\hline \multicolumn{5}{|l|}{ Suicídios } \\
\hline 2013 & 4,05 & 6,72 & 4,47 & 5,24 \\
\hline 2017 & 7,78 & 7,14 & 4,96 & 5,7 \\
\hline \multicolumn{5}{|c|}{ Homicídios, incluindo intervenções } \\
\hline 2013 & 44,01 & 50,95 & 39,72 & 28,55 \\
\hline 2017 & 58,39 & 60,07 & 47,31 & 30,45 \\
\hline \multicolumn{5}{|c|}{ Causas de intenção indeterminada } \\
\hline 2013 & 0,51 & 3,83 & 5,59 & 4,87 \\
\hline 2017 & 0 & 5,59 & 7,12 & 6,49 \\
\hline \multicolumn{5}{|l|}{ Outras causas } \\
\hline 2013 & 17,2 & 13,98 & 12,94 & 15,18 \\
\hline 2017 & 14,6 & 13,39 & 13,22 & 15,61 \\
\hline
\end{tabular}

Fonte: Rios Junior WO, et al., 2020. Dados extraídos do Sistema de Informações sobre Mortalidade (SIM). 
Em relação à taxa de mortalidade por acidentes de transportes, observa-se uma redução considerável em todas as esferas. No município de sobral essa taxa caiu de 33,39 para 26,76 óbitos por 100 mil habitantes, representando uma redução de $19,86 \%$ de casos. Mesmo com essa redução, os valores continuam acima dos encontrados nas esferas estadual, regional e nacional.

A redução de óbitos por acidentes de transporte também foi encontrada em estudos realizados na Região Sul, com declínio importante no ano de 2004 para 2013 (PREIS LC, et al., 2018), e em Curitiba-PR, de 1996 a 2011 (BARRETO MS, et al., 2016).

No que se refere à taxa de mortalidade por homicídios, Sobral fica atrás apenas no âmbito estadual, apresentando nos anos de 2013 e 2017, taxas de 44,01 e 58,39 óbitos por 100 mil habitantes, números superiores aos encontrados em âmbito regional e nacional. Em todas as esferas, os homicídios, incluindo as intervenções legais, representam a principal causa de mortalidade por causas externas.

Já em relação aos casos de suicídios, o município apresenta uma inversão ao longo dos anos, saindo do menor valor ao se comparar as três esferas $(4,05)$, ao valor mais alto $(7,78)$, representando um aumento da taxa de mortalidade em $92,1 \%$. O aumento de número de óbitos por lesões autoprovocadas intencionalmente também foi encontrado em pesquisa realizada na Região Sul, de 2004 a 2013.

Houve predomínio de óbitos em motociclistas, com um total de 139 casos no período analisado, que correspondem a $49,12 \%$ dos óbitos por acidentes de transportes e $13,91 \%$ dos óbitos por causas externas. O número de óbitos de pedestre superou, no ano de 2017, o de óbitos de indivíduos em automóveis, assumindo a segunda posição em prevalência, com 15,55\% dos casos (Gráfico 2).

Gráfico 2 - Perfil dos óbitos por acidentes de transporte em Sobral, CE no período de 2013 a 2017 (número absoluto).

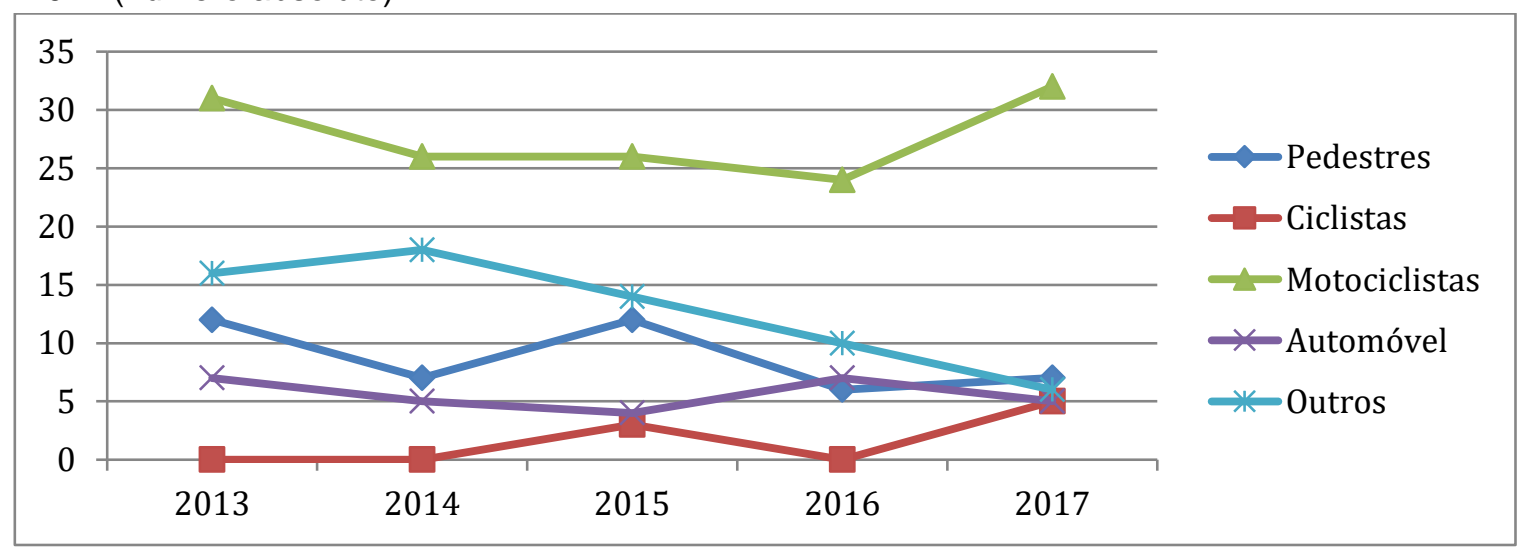

Fonte: Rios Junior WO, et al., 2020. Dados extraídos do Sistema de Informações sobre Mortalidade (SIM).

A motocicleta é o meio de transporte mais citados em artigos que caracterizam o perfil dos acidentes de trânsito. Acredita-se que isso se deve fato de que as motocicletas estão sendo cada vez mais utilizadas no país, por ser proporcionar um transporte rápido e de baixo custo, tanto para aquisição quando para manutenção, sendo utilizado, muitas vezes, como instrumento de trabalho (MELO GBT, et al., 2015). Além disso, no município de Sobral, um dos principais meios de deslocamento da população é feito através do transporte por mototaxistas.

Em relação à faixa etária das vítimas de acidentes de transportes, observou-se que a maior quantidade de óbitos ocorreu entre os jovens de 20 a 39 anos, com 136 (48,06\%) casos, seguido dos indivíduos com idade entre 40 e 59 anos (31,09\%). Entre os idosos, o número de casos foi de 39 , representando $13,78 \%$ dos casos de óbitos por acidentes de transporte.

No que diz respeito ao sexo desses indivíduos, houve maioria expressiva no número de óbitos masculinos, com $238(84,1 \%)$ casos, contra apenas $45(15,9 \%)$ óbitos femininos em acidentes de transporte. Já em relação à cor/raça, o predomínio foi de pardos, com 232 (81,98\%) óbitos, seguido pelo de brancos (15,9\%). 
Houve predomínio no número absoluto de homicídios com arma de fogo, bem como o aumento expressivo de número de casos no ano de 2017. No período analisado, ocorreram 370 casos de homicídios por esse meio, o que corresponde a $75,2 \%$ dos óbitos por agressão e $37,04 \%$ dos óbitos por causas externas. $17,89 \%$ dos homicídios tiveram com meio de agressão o uso de armas brancas, enquanto em $6,09 \%$ foram empregados outros meios (Gráfico 3).

Gráfico 3 - Meios empregados nos óbitos por homicídios em Sobral, CE no período de 2013 a 2017 (número absoluto).

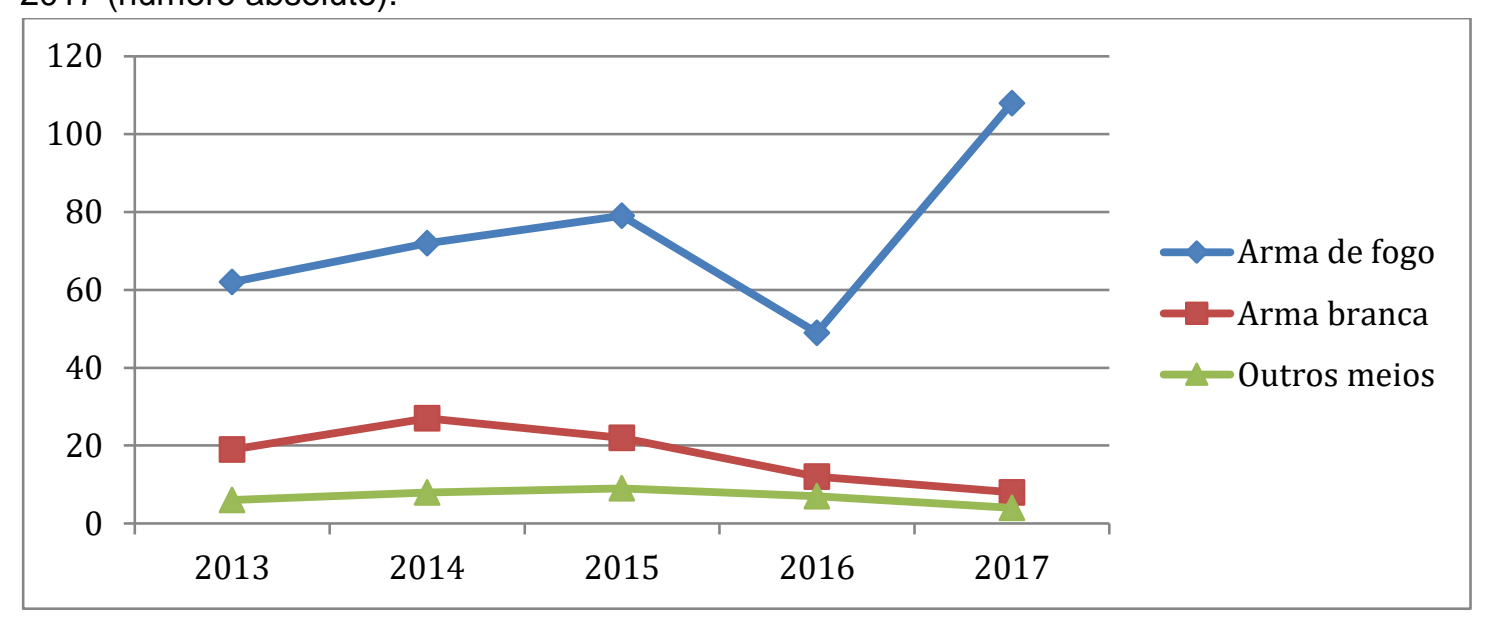

Fonte: Rios Junior WO, et al., 2020. Dados extraídos do Sistema de Informações sobre Mortalidade (SIM).

O homicídio com arma de fogo foi o principal fator de mortalidade por causas externas evidenciado em revisão de literatura realizada em 2016 no Brasil, com crescimento progressivo desde a década de 80 (MELO AUC, 2016). Ao analisar a faixa etária das vítimas de homicídio, houve predomínio de jovens entre 20 e 39 anos, com 286 (58,13\%), seguida pelo faixa etária de 15 a 19 anos, com 121 (24,59\%) casos. Em relação aos idosos, houveram apenas $9(1,83 \%)$ casos.

Mais uma vez, os óbitos entre o sexo masculino prevalecem, neste caso, com porcentagem ainda mais expressiva. 460 homens foram vítimas de homicídio no período de 2013 a 2017 no município de Sobral, equivalente a $93,5 \%$ de casos de óbitos por agressões. A situação se repete também quando a cor/raça dos indivíduos é analisada. 92,3\% dos óbitos por homicídio foram de pardos, enquanto apenas 5,5\%, de brancos. Reforçando o perfil predominante das vítimas de causas externas no município analisado.

Por fim, em relação aos casos de suicídio, houve predomínio em jovens de 20 a 39 anos, com 24 (35,29\%) casos no período analisado, em indivíduos do sexo masculino, com 56 (82,35\%) óbitos e em pardos, com 62 $(91,18 \%)$ por lesões autoprovocadas intencionalmente. Esse perfil também foi encontrado em estudo realizado no Brasil, entre 1980 e 2006 (LOVISI GM, et al., 2009). Acredita-se que o sexo masculino seja o grupo mais acometido em razões de fatores comportamentais, como competitividade, impulsividade e maior acesso a tecnologias letais e armas de fogo, bem como emocionais, sendo mais sensíveis a instabilidades econômicas como desemprego e empobrecimento (PARENTE ACM, 2009).

Em relação à faixa etária, o aumento no número de suicídios em jovens pode estar relacionado à grande pressão ocupacional e aumento da competitividade no mercado de trabalho (SOUZA ER, et al., 2002), sendo este resultado encontrado, também, em estudo realizado em Teresina-PI, de 2000 a 2005 (PARENTE ACM, 2009).

\section{CONCLUSÃO}

Através desse estudo, tornou-se possível observar o progressivo aumento do número de mortes decorrentes de causas externas no município de Sobral. A população mais acometida pertencia aos grupos 
masculino, com idade entre 20 e 39 anos e de cor parda. Observou-se, ainda, o aumento das taxas de mortalidade, no período analisado, em todos os subgrupos, com exceção dos óbitos relacionados aos acidentes de trânsito. Os resultados reforçam a necessidade do desenvolvimento não apenas de políticas de saúde, mas também políticas sociais e de segurança pública, visto que o maior número de vítimas apresenta o perfil daqueles marginalizados pela sociedade: o jovem pardo de baixo nível socioeconômico. Reforça, ainda, a necessidade de medidas de apoio psicológico aos jovens, grupo mais acometido por mortalidade por lesões autoprovocadas.

\section{REFERÊNCIAS}

1. ASCARI RA, et al. Perfil epidemiológico de vítimas de acidente de trânsito. Rev Enferm UFSM, 2013; 3(1):112-121.

2. BARRETO MS, et al. Mortalidade por acidentes de trânsito e homicídios em Curitiba, Paraná, 1996-2011. Epidemiol. Serv. Saúde, 2016; 25 (1): 95-104.

3. BRASIL. Viva: Vigilância de Violências e Acidentes: 2013 e 2014 [recurso eletrônico] / Ministério da Saúde, Secretaria de Vigilância em Saúde, Departamento de Vigilância de Doenças e Agravos Não Transmissíveis e Promoção da Saúde. - Brasília: Ministério da Saúde, 2017. Disponível em: <http://bvsms.saude.gov.br/bvs/publicacoes/viva_2013_2014.pdf>. Acesso em 14 mai. 2019

4. CORASSA RB, et al. Evolução da mortalidade por causas externas em Diamantina (MG), 2001 a 2012. Cad. saúde colet, 2017; 25 (3): 302-314.

5. GONSAGA RAT, et al. Avaliação da mortalidade por causas externas. Rev. Col. Bras. Cir, 2012; 39(4): 263-267

6. LIMA TF, el al. Análise epidemiológica dos acidentes de trânsito no Brasil. Encontro de Extensão, Docência e Iniciação Científica, 2019; 5 (1): 9-16.

7. LOPE AS, et al. Melhoria da qualidade do registro da causa básica de morte por causas externas a partir do relacionamento de dados dos setores Saúde, Segurança Pública e imprensa, no estado do Rio de Janeiro, 2014. Epidemiol. Serv. Saúde, 2018; 27 (4): 1-10.

8. LOVISI GM, et al. Análise epidemiológica do suicídio no Brasil entre 1980 e 2006. Revista Brasileira de Psiquiatria, 2009; 31(20): 86-93.

9. MELO AUC, et al. Perfil epidemiológico da mortalidade por causas externas: uma análise da literatura no brasil. Revista de Saúde UniAGES, 2016; 1 (1): 9-32.

10. MELO GBT, et al. Mortalidade causas externas em Pernambuco,2001-2003 e 2011-2013. Rev Bras Enferm. 2015; 68(5):573-579.

11. MESSIAS MM, et al. Mortalidade por causas externas: revisão dos dados do Sistema de Informação de Mortalidade. Rev Soc Bras Clin Med, 2018;16(4):218-21

12. MODESTO JB, et al. Fatores que influenciam na mortalidade de jovens por causas externas no Brasil: uma revisão da literatura. Revista Multidebates, 2019; 3 (2): 137-155.

13. MOURA EC, et al. Desigualdades de gênero na mortalidade por causas externas no Brasil, 2010. Ciência \& Saúde Coletiva, 2015; 20(3):779-788.

14. PARENTE ACM. Caracterização dos casos de suicídio em uma capital do Nordeste brasileiro. Rev Bras Enferm, 2007; 60(4): 377-381.

15. PREIS LC, et al. Epidemiologia da mortalidade por causas externas no período de 2004 a 2013. Rev enferm UFPE, 2018; 12(3):716-28.

16. SILVA, BFA, et al. O suicídio no Brasil contemporâneo. Soc. estado., 2018; 33, (2): 565-579.

17. SOUZA ER, et al. Suicídio de jovens nas principais capitais do Brasil. Cad. Saúde Pública [online]. $2002 ; 18$ (3): $673-$ 683.

18. TAVARES J, et al. Transição epidemiológica e causas externas de mortalidade na região sudeste do Brasil. Revista de Geografia e Ordenamento do Território, 2018; 15 (1): 453-479.

19. VASCONCELOS SM. O impacto da lei seca na redução dos acidentes de transporte terrestre em um município do nordeste brasileiro. Dissertação (Mestrado em Gestão e Economia da Saúde) - Centro de Ciências Sociais Aplicadas. Universidade Federal de Pernambuco, Recife, 2015; 27p.

20. WORLD HEALTH ORGANIZATION, 2018. The top 10 causes of death. 2018. Geneva: WHO. Disponível em: <https://www.who.int/news-room/fact-sheets/detail/the-top-10-causes-of-death>. Acesso em 25 mai. 2020. 